\title{
A Sonochemical Route to Single-walled Carbon Nanotubes under Ambient Conditions
}

\author{
Soo-Hwan Jeong, $†$ Ju-Hye Ko,‡ Jong-Bong Park,§ Wanjun Park $¥$
}

U-team, MD Lab and AE center, Samsung Advanced Institute of Technology (SAIT), P. O. Box 111, Suwon 440-600, Korea,

\section{Supporting Information}
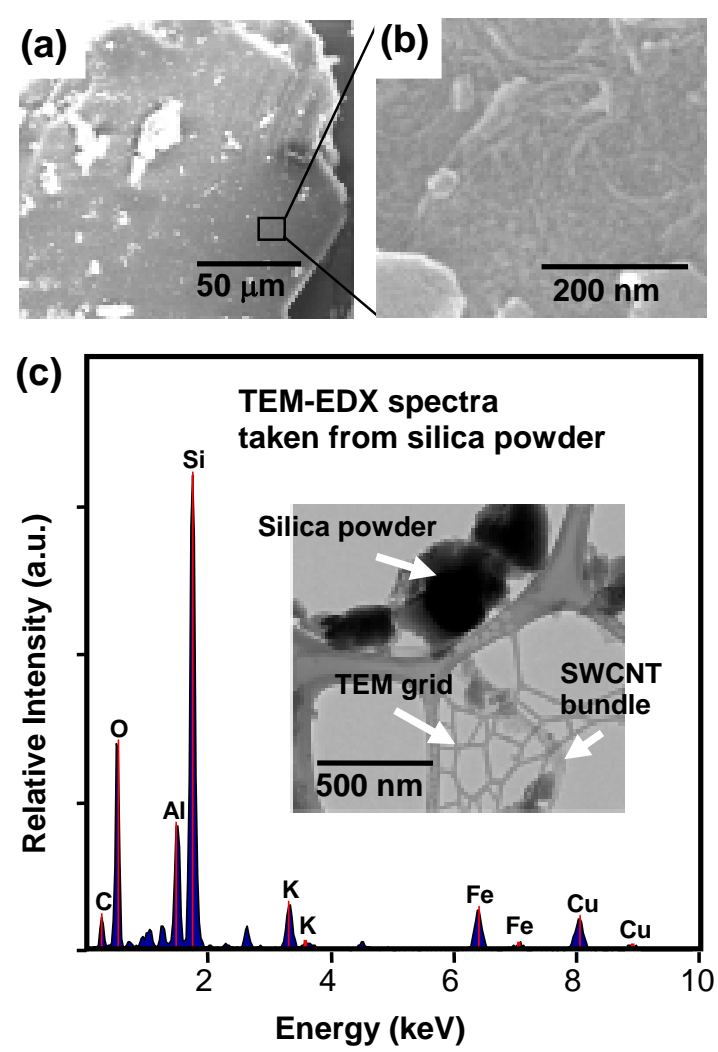

Figure S1. FESEM images and TEM-EDX spectra of samples after sonication treatment. (a) FESEM image of silica powder (low magnification), (b) enlarged view of selected area in (a). (c) typical TEM-EDX spectra of silica powder, which is shown in inset of (c). Inset is low-resolution TEM image of sample for EDX spectra. Samples were corrected on alumina membrane after sonication treatment without removing silica powder. The peak of Fe $(\mathrm{K} 1=6.40$ $\mathrm{eV})$ is considerable, which confirms the formation of Fe catalyst. The peaks of $\mathrm{Si}$ and $\mathrm{O}$ resulted from silica powder. Cu peak could be attributed to the $\mathrm{Cu}$ element in TEM grid. It is also expected that $\mathrm{Al}$ and $\mathrm{K}$ peaks resulted from alumina filter membrane. 


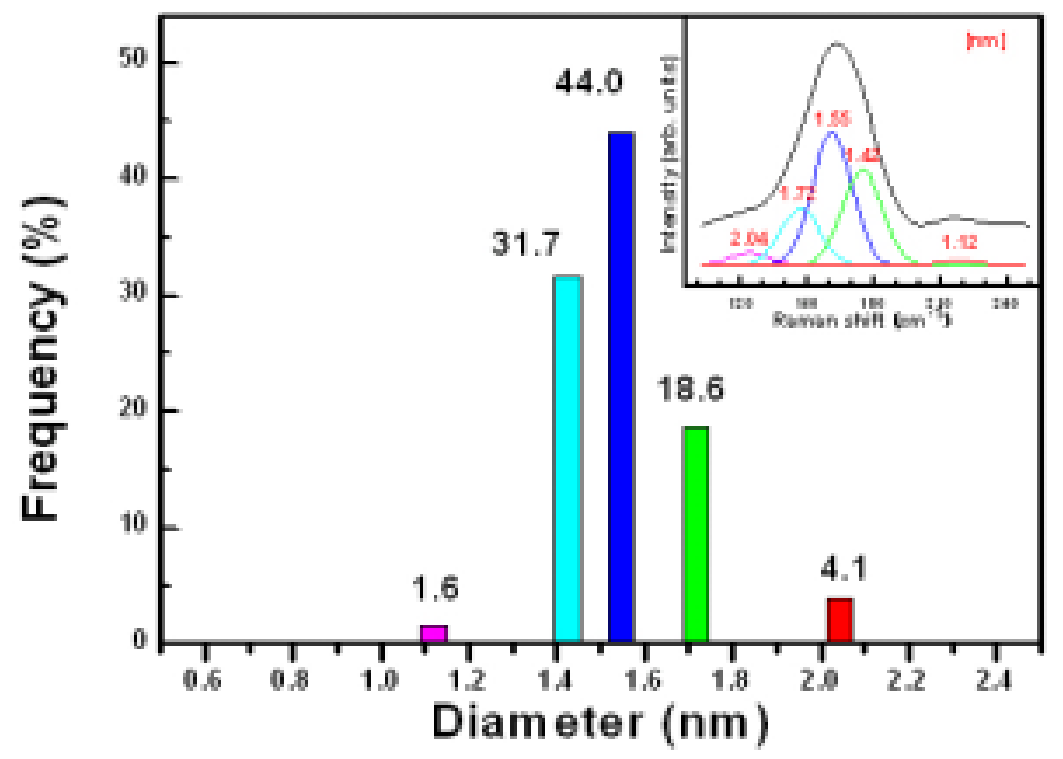

Figure S2. Diameter distribution of grown SWCNTs. The data are calculated from Raman spectra (Ar+ laser, $\lambda=514.5 \mathrm{~nm}, 25 \mathrm{~mW}$ ). Lorentzian curves fitted to Raman RBM data are also presented (inset). 